\title{
Verzeichnis der Bearbeiter der 13. Auflage
}

Dr. Philipp Ambach, Chief, Victim Participation and Reparations, Section Registry, International Criminal Court

Gerhard Altvater, Bundesanwalt beim Bundesgerichtshof (Abteilungsleiter) a.D., Karlsruhe

Elisabeth Baier, LL.M., Rechtsanwältin, Berlin

Dr. Christoph Barthe, Oberstaatsanwalt beim Bundesgerichtshof, Richter am Sondergerichtshof für den Kosovo (Kosovo Specialist Chambers)

Dr. Alexander Baur, Juniorprofessor an der Universität Hamburg

Dr. Christian Brand, Universität Konstanz

Dr. Dominik Brodowski, LL.M. (UPenn), Juniorprofessor an der Universität des Saarlandes

Dr. Christoph Burchard, LL.M., Universitätsprofessor an der Goethe-Universität Frankfurt am Main

Dr. Jens Bülte, Universitätsprofessor an der Universität Mannheim

Gabriele Cirener, Vorsitzende Richterin am Bundesgerichtshof, Leipzig

Dr. Christoph Coen, Oberstaatsanwalt beim Bundesgerichtshof, Karlsruhe

Dr. Dr. h.c. Gerhard Dannecker, Seniorprofessor an der Ruprecht-Karls-Universität Heidelberg

Dr. Tobias Engelstätter, Oberstaatsanwalt beim Bundesgerichtshof, Karlsruhe

Dr. Robert Esser, Universitätsprofessor an der Universität Passau

Dr. Julia Gebhard, Legislative Support Officer, OSZE Büro für Demokratische Institutionen und Menschenrechte (OSZE/ODIHR)

Dr. Oliver Harry Gerson, Universität Passau

Dr. Ferdinand Gillmeister, Rechtsanwalt, Freiburg, Honorarprofessor an der Universität Bayreuth

Dr. Ingke Goeckenjan, Universitätsprofessorin an der Ruhr-Universität Bochum

Dr. Luís Greco, LL.M., Universitätsprofessor an der Humboldt-Universität zu Berlin

Anette Greger, Oberstaatsanwältin beim Bundesgerichtshof, Karlsruhe

Dr. Andreas Grube, Richter am Bundesgerichtshof, Karlsruhe

Dr. Anette Grünewald, Universitätsprofessorin an der Friedrich-Schiller-Universität Jena

Dr. Georg-Friedrich Güntge, Leitender Oberstaatsanwalt bei der Generalstaatsanwaltschaft in Schleswig, Honorarprofessor an der Christian-Albrechts-Universität zu Kiel

Dr. Michael Heghmanns, Universitätsprofessor an der Westfälischen Wilhelms-Universität Münster, Vorsitzender Richter am Landgericht Münster

Gregor Herb, Vorsitzender Richter am Landgericht Berlin

Dr. Mayeul Hiéramente, Rechtsanwalt (Fachanwalt für Strafrecht), Hamburg

Dr. Dr. Eric Hilgendorf, Universitätsprofessor an der Julius-Maximilians-Universität Würzburg

Dr. Tatjana Hörnle, Direktorin des MPI zur Erforschung von Kriminalität, Sicherheit und Recht, Freiburg i.Brsg., Honorarprofessorin an der Humboldt-Universität zu Berlin

Dr. Kristian Hohn, Privatdozent an der Bucerius Law School Hamburg

Dr. Jutta Hubrach, Vorsitzende Richterin am Oberlandesgericht Düsseldorf

Dr. Florian Jeßberger, Universitätsprofessor an der Humboldt-Universität zu Berlin

Dr. Johannes Koranyi, Richter am Landgericht Bonn

Dr. Peter König, Richter am Bundesgerichtshof, Leipzig, Honorarprofessor an der Ludwig-Maximilians-Universität München

Dr. Ralf Krack, Universitätsprofessor an der Universität Osnabrück

Juliane Krause, Richterin am Bayerischen Obersten Landesgericht, Bamberg

Dr. Matthias Krauß, Bundesanwalt beim Bundesgerichtshof, Karlsruhe

Dr. Christoph Krehl, Richter am Bundesgerichtshof, Karlsruhe, Honorarprofessor an der Goethe-Universität Frankfurt am Main

Helena Krüger, Wissenschaftliche Mitarbeiterin an der Universität Passau

Dr. Matthias Krüger, Universitätsprofessor an der Universität München

Dr. Dr. h.c. Michael Kubiciel, Universitätsprofessor an der Universität Augsburg 
Dr. Hans Kudlich, Universitätsprofessor an der Friedrich-Alexander-Universität Erlangen-Nürnberg

Stefanie Küfner, Legal \& Policy Officer, HRS, Registry, International Criminal CourtDr. Michael Lindemann, Universitätsprofessor an der Universität Bielefeld

Dr. Alexander Linke, Richter am Landgericht Köln

Kai Lohse, Bundesanwalt beim Bundesgerichtshof, Karlsruhe

Dr. Manfred Möhrenschlager, Ministerialrat a.D., Bonn

Dr. Andreas Mosbacher, Richter am Bundesgerichtshof, Leipzig, Honorarprofessor an der Universität Leipzig

Dr. Svenja Münzner, Lehrbeauftragte an der Justus-Liebig-Universität Gießen, Staatsanwältin beim Bundesgerichtshof, Karlsruhe

Dr. Uwe Murmann, Universitätsprofessor an der Georg-August-Universität Göttingen

Dr. Nina Nestler, Universitätsprofessorin an der Universität Bayreuth

Dr. Jens Peglau, Richter am Oberlandesgericht, Hamm

Dr. Andreas Popp, M.A., Universitätsprofessor an der Universität Konstanz

Dr. Henning Radtke, Richter des Bundesverfassungsgerichts, Karlsruhe, Honorarprofessor an der Leibniz Universität Hannover

Dr. Ruth Rissing-van Saan, Vorsitzende Richterin am Bundesgerichtshof a.D., Bochum, Honorarprofessorin an der Ruhr-Universität Bochum

Dr. Thomas Rönnau, Universitätsprofessor an der Bucerius Law School Hamburg

Dr. Henning Rosenau, Universitätsprofessor an der Martin-Luther-Universität Halle-Wittenberg

Dr. h.c. Wilhelm Schluckebier, Richter des Bundesverfassungsgerichts a.D., Karlsruhe

Dr. Wilhelm Schmidt, Bundesanwalt beim Bundesgerichtshof a.D., Karlsruhe

Dr. Ursula Schneider, Richterin am Bundesgerichtshof, Leipzig

Daniel Scholze, wissenschaftlicher Mitarbeiter am Bundesgerichtshof, Karlsruhe

Dr. Dres. h.c. Friedrich-Christian Schroeder, em. Universitätsprofessor an der Universität Regensburg

Dr. Dr. h.c. mult. Bernd Schünemann, em. Universitätsprofessor an der Ludwig-MaximiliansUniversität München

Dr. Jan C. Schuhr, Universitätsprofessor an der Ruprecht-Karls-Universität Heidelberg

Dr. Christoph Sowada, Universitätsprofessor an der Universität Greifswald

Dr. Mark Steinsiek, Ministerialrat, Niedersächsisches Ministerium für Wirtschaft, Arbeit, Verkehr und Digitalisierung

Dr. Brian Valerius, Universitätsprofessor an der Universität Bayreuth

Dr. Torsten Verrel, Universitätsprofessor an der Universität Bonn

Dr. Dr. Dr. h.c. Thomas Vormbaum, Universitätsprofessor an der Fern-Universität in Hagen

Dr. Tonio Walter, Universitätsprofessor an der Universität Regensburg, Richter am Bayerischen Obersten Landesgericht

Dr. Thomas Weigend, em. Universitätsprofessor an der Universität zu Köln

Jochen Weingarten, Oberstaatsanwalt beim Bundesgerichtshof, Karlsruhe

Lienhard Weiß, Bundesanwalt beim Bundesgerichtshof, Karlsruhe

Dr. Gerhard Werle, Universitätsprofessor an der Humboldt-Universität zu Berlin

Stefan Wiedner, Richter am Oberlandesgericht Koblenz

Dr. Gereon Wolters, Universitätsprofessor an der Ruhr-Universität Bochum, Mitglied des Verfassungsgerichtshofes für das Land Nordrhein-Westfalen

Dr. Frank Zieschang, Universitätsprofessor an der Julius-Maximilians-Universität Würzburg

Dr. Georg Zimmermann, Vorsitzender Richter am Landgericht Bielefeld

Kathrin Zitzelsberger, Universität Passau 